\section{Global Anisotropies in TeV Cosmic Rays Related to the Sun's Local Galactic Environment from IBEX}

\author{
N. A. Schwadron, ${ }^{1,2 *}$ F. C. Adams, ${ }^{3}$ E. R. Christian, ${ }^{4}$ P. Desiati, ${ }^{5}$ P. Frisch, ${ }^{6}$ H. O. Funsten, \\ J. R. Jokipii, ${ }^{8}$ D. J. McComas, ${ }^{2,9}$ E. Moebius, ${ }^{1}$ G. P. Zank ${ }^{10}$
}

Observations with the Interstellar Boundary Explorer (IBEX) have shown enhanced energetic neutral atom (ENA) emission from a narrow, circular ribbon likely centered on the direction of the local interstellar medium (LISM) magnetic field. Here, we show that recent determinations of the local interstellar velocity, based on interstellar atom measurements with IBEX, are consistent with the interstellar modulation of high-energy (tera-electron volts, $\mathrm{TeV}$ ) cosmic rays and diffusive propagation from supernova sources revealed in global anisotropy maps of ground-based high-energy cosmic-ray observatories (Milagro, As $\gamma$, and IceCube). Establishing a consistent local interstellar magnetic field direction using IBEX ENAs at hundreds to thousands of eV and galactic cosmic rays at tens of TeV has wide-ranging implications for the structure of our heliosphere and its interactions with the LISM, which is particularly important at the time when the Voyager spacecraft are leaving our heliosphere.

$\mathrm{T}$ he heliosphere - the region surrounding our solar system that is carved out by the supersonic solar wind-moves through the local interstellar cloud (LIC), which is the part of the galactic environment surrounding the Sun. The heliosphere deflects much of the harmful cosmic-ray radiation from the local galactic environment, thereby regulating the radiation environment throughout the solar system, which likely has important implications for Earth's atmosphere and life (1). The Interstellar Boundary Explorer (IBEX) recently provided updated values for the velocity vector of the heliosphere through the LIC (2) and direction for the local interstellar medium (LISM) magnetic field (Table 1 and Fig. 1) from the center of the IBEX ribbon of energetic neutral atom (ENA) emission (3-9). These results show that the interstellar flow, after removing the motion of the Sun through the local standard of rest (LSR), is nearly perpendicular $\left(87.6 \pm 3.0^{\circ}\right)$ to the LISM magnetic field. The LSR describes the velocity frame in which the mean motion of the oldest stars in the Milky Way in the neighborhood of the Sun is zero and is the reference frame in which cosmic rays assume near uniformity (in velocity direction). The local interstellar magnetic field direction from the IBEX ribbon center is

${ }^{1}$ University of New Hampshire, Space Science Center, Durham, NH 03824, USA. ${ }^{2}$ Southwest Research Institute, San Antonio, TX 78228, USA. ${ }^{3}$ University of Michigan, Physics Department, Ann Arbor, MI 48109, USA. ${ }^{4}$ Goddard Space Flight Center, Greenbelt, MD 20771, USA. ${ }^{5}$ University of Wisconsin, IceCube Observatory and Astronomy Department, Madison, WI 53706, USA. 'University of Chicago, Department of Astronomy and Astrophysics, Chicago, IL 60637, USA. 'Los Alamos National Laboratory, Space Science and Applications, Los Alamos, NM 87545, USA. ${ }^{8}$ University of Arizona, Department of Planetary Sciences, Tucson, AZ 85721, USA. 'University of Texas at San Antonio, San Antonio, TX 78249, USA. ${ }^{10}$ University of Alabama, Huntsville, AL 35805, USA.

*Corresponding author. E-mail: n.schwadron@unh.edu

within $\sim 33 \pm 20^{\circ}$ of the magnetic field direction derived by interstellar polarization data from stars within $40 \mathrm{pc}(10,11)$. Here, we show that the anisotropy maps of high-energy $(\mathrm{TeV})$ cosmic rays likely provide independent confirmation of the interstellar magnetic field orientation inferred from the IBEX ribbon center.

The flux of high-energy (TeV) galactic cosmic rays (GCRs) varies as a function of look direction in the sky. The large-scale structure in the TeV GCR sky consists of two broad asymmetries with flux variations of $\sim 0.2 \%$ : a deficit of GCR flux at high galactic latitudes (the "loss cone") and an excess of flux in the heliotail (12) direction [the "tail-in" excess (13-21)]. Small-scale $\mathrm{TeV}$ anisotropies $\left(<\sim 10^{\circ}\right)$ in cosmic-ray arrival directions possibly arise from cosmic-ray propagation in a turbulent magnetic field (22). Because TeV GCRs have gyroradii of $<700$ astronomical units (AUs) $(<0.005$ parsec or pc; $1 \mathrm{pc}=3 \times$
Fig. 1. LIC velocities and LISM magnetic field directions. The LIC velocities inferred from IBEX (2) in the heliocentric (HC; green vector) rest frame and the local standard of rest (LSR; black vector) in the galaxy. These vectors are shown in both the galactic plane (lower plane) and the plane containing the galactic poles (upper plane) relative to the magnetic field of the local interstellar medium (LISM; blue lines). The LIC velocity and magnetic field are a part of the Loop I superbubble roughly centered on the Lower Centaurus Crux subgroup of the Scorpius-Centaurus Association. The cosmic-ray anisotropy (gray vector) indicates average streaming of cosmic rays. $\left.10^{18} \mathrm{~cm}=200,000 \mathrm{AU}\right)$ in the LISM, the observed GCR asymmetries must originate in the immediate interstellar environment of the Sun. This study extends and builds on increasing knowledge of the LISM magnetic field $(3,4,10,11,23,24)$ and interstellar flow $(2,25-28)$ to understand the origin of the large-scale high-energy (TeV) cosmicray anisotropy, which has remained uncertain.

The observed cosmic-ray anisotropy has a number of features that may reflect ordering both by the entry of cosmic rays into the heliosphere and by the interstellar magnetic field. It has been found (13) that the tail-in excess weakens above $10 \mathrm{TeV}$ because of the relative sizes of the GCR gyroradius and heliotail. Data from underground detectors in both hemispheres has led to precise positions of the asymmetries (14). Additionally, the underground muon observatory Super-Kamiokande I obtained similar asymmetries for the $10-\mathrm{TeV}$ GCR flux, with a deficit toward the constellation of Virgo representing the northern galactic deficit, and an excess in the direction of the heliotail, toward the constellation of Taurus (15). The direction of the Taurus excess is within $29^{\circ}$ of the local interstellar magnetic field defined by the IBEX ribbon (Table 1) and within $23^{\circ}$ from the local interstellar downwind direction (2). Milagro observations in the Northern Hemisphere (19), in combination with IceCube observations in the Southern Hemisphere (20), have confirmed that the galactic deficit is centered at high galactic latitudes and extends into both hemispheres (21). A global GCR anisotropy model constructed with unidirectional and bidirectional components and applied to results of the Tibet Asy experiment attributed the bidirectional flow to GCRs drifting parallel to the local interstellar magnetic field.

The cosmic-ray anisotropy, according to standard diffusion theory [e.g., $(29,30)]$, is proportional to the average streaming of cosmic rays. Here, we take a small $[0.3 \%(31)]$ ratio of perpendicular to parallel diffusion, as well as an interstellar flow direction to be nearly perpendicular to the magnetic field on the basis of IBEX observations.

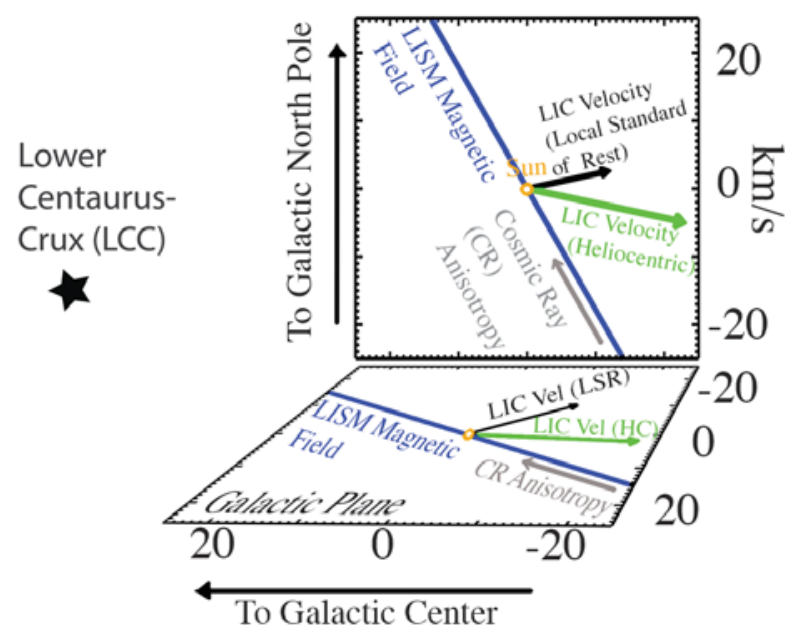


In this parameter regime, cosmic rays are largely guided by the interstellar magnetic field because both the anisotropic and perpendicular components of the anisotropy (fig. S1) are small compared to the field-aligned component.

We consider two scenarios for the formation of the cosmic-ray anisotropy. In the first scenario, the magnitude of the anisotropy is determined in part by spatial gradients in the average cosmicray density in response to the interstellar flow (eq. S6 in supplementary text). For example, an outward plasma flow from the center of the Lower Centaurus Crux (LCC) cluster (Fig. 1) pushes vironment in which the heliosphere resides. This creates a spatial gradient in the cosmic-ray density in the direction of the source and flow, which the average streaming (gray vector, Fig. 1) of cosmic rays tends to oppose.

The streaming direction of cosmic rays is determined on relatively small scales (on the scale of the cosmic-ray gyroradii, less than $1 \%$ of a pc), whereas the streaming magnitude is determined over much larger scales of tens of pc $(32,33)$ some cosmic rays out of the local interstellar en-

controlled by the scattering (random changes in direction) of cosmic rays caused by interactions with the turbulent interstellar magnetic field.

It is important to differentiate $(34,35)$ between the interstellar magnetic field on local scales (thousands of $\mathrm{AU}$ or $\sim 0.005$ to $0.01 \mathrm{pc}$ ) and on larger (parsec) scales owing to the presence of turbulence (36-41). Turbulence disrupts steady flows by random motion. In the interstellar medium, turbulent motion causes tangling and complexity in the structure of the interstellar magnetic field. The coherence scale length in the interstellar medium - the approximate distance along which the magnetic field appears relatively ordered - is typically 1 to $10 \mathrm{pc}$ (37). Hence, the average magnetic field direction at these scales could be very different than in the vicinity of the heliosphere. At the large field-to-flow angle (87.6 \pm $3.0^{\circ}$ ) found by IBEX (excepting the small region within $0.3^{\circ}$ of exact perpendicularity, where perpendicular diffusion dominates for the $0.3 \%$ ratio of perpendicular to parallel diffusion taken here), the cosmic-ray density gradient becomes large so that the projection of the cosmic-ray streaming

Table 1. LISM velocity and magnetic field direction.

\begin{tabular}{lccccr} 
& $\begin{array}{c}\text { Magnitude } \\
(\mathrm{km} / \mathrm{s})\end{array}$ & $\begin{array}{c}\text { Galactic } \\
\text { longitude }\left(^{\circ}\right)\end{array}$ & $\begin{array}{c}\text { Galactic } \\
\text { latitude }\left({ }^{\circ}\right)\end{array}$ & $\begin{array}{c}\text { Equatorial right } \\
\text { ascension }\left(^{\circ}\right)\end{array}$ & $\begin{array}{c}\text { Equatorial } \\
\text { declination }\left(^{\circ}\right)\end{array}$ \\
\hline $\begin{array}{l}\text { LISM flow velocity* } \\
(\text { HC frame) }\end{array}$ & $23.2 \pm 0.3$ & $185.25 \pm 0.24$ & $-12.0 \pm 0.5$ & $78.5 \pm 0.6$ & $18.0 \pm 0.5$ \\
$\begin{array}{l}\text { LISM flow velocity } \\
\quad\left(\text { LSR frame }{ }^{\dagger}\right)\end{array}$ & $18.0 \pm 0.9$ & $47.9 \pm 2.9$ & $23.8 \pm 2.0$ & $267.0 \pm 3.0$ & $23.2 \pm 3.1$ \\
$\begin{array}{l}\text { Interstellar magnetic } \\
\text { field }\end{array}$ & & $210.5 \pm 2.6$ & $-57.1 \pm 1.0$ & $48.5 \pm 1.5$ & $-21.2 \pm 1.6$ \\
\hline
\end{tabular}

*Heliocentric (HC) rest frame (2). H Heliocentric velocities are converted to the local standard of rest (LSR) using the solar apex motion in (54). ‡Field directions and uncertainty inferred from the IBEX highest-energy steps [1.79 and 2.73 keV (55)] in which the ribbon maintains coherence and has the largest line-of-sight.

\section{Observed}

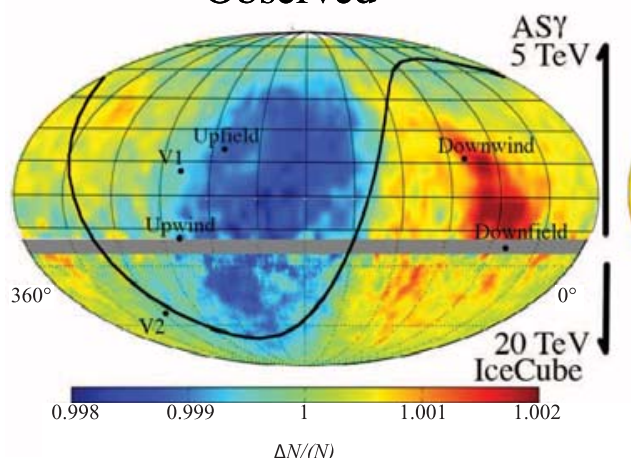

$\Delta N /(N)$
Interstellar Conditions from IBEX

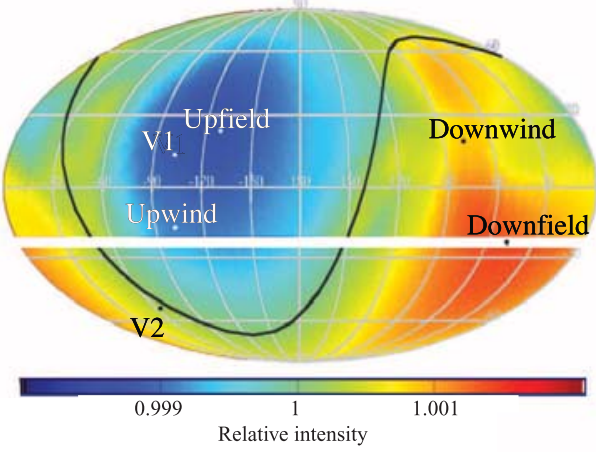

Fig. 2. TeV cosmic-ray anisotropies compared with predictions. Comparison between observed (left) and modeled (right) cosmic-ray relative intensities across the sky (J2000 coordinates). Black curves show the magnetic equator with a magnetic field direction derived from the center of the IBEX ribbon. On the left, the region below $25^{\circ} \mathrm{S}$ latitude is the anisotropy map from IceCube with a median energy of $20 \mathrm{TeV}(18)$ and above $20^{\circ} \mathrm{S}$ latitude is the anisotropy map from AS $\gamma$ with $5-\mathrm{TeV}$ median energy (15). Similarly, the modeled map (right) at $20 \mathrm{TeV}$ is shown below $25^{\circ} \mathrm{S}$ latitude and at $5 \mathrm{TeV}$ above $20^{\circ} \mathrm{S}$ latitude. Both portions of the maps are smoothed over $3^{\circ}$ to $5^{\circ}$. Labels indicate upwind and downwind directions (2), the current locations of Voyager 1 (V1), and Voyager 2 (V2) directions, and the "upfield" and "downfield" directions. Downfield is along the LISM magnetic field determined by IBEX in the direction closest to the interstellar velocity, and upfield is in the opposite direction. Plots are in equatorial coordinates with 0 hours at the right and increasing longitudes toward the left. into the flow plane opposes the interstellar velocity. The cosmic-ray gradient in this case depends largely on the ratio of perpendicular to parallel diffusion. Reduced levels of perpendicular diffusion cause increased density gradients and thus stronger interstellar modulation of cosmic rays.

The $\sim 0.2 \%$ high-energy (TeV) cosmic-ray anisotropy suggests a magnitude for the ratio of perpendicular to parallel diffusion of only $0.3 \%$ (fig. S1). This ratio is compatible with recent results (31), but an order-of-magnitude smaller than the derived $\sim 4 \%$ ratio of perpendicular to parallel diffusion (41) based on cosmic-ray lifetimes in the galaxy (39). Simulations of cosmic-ray diffusion (42) similarly predicted a ratio of perpendicular to parallel diffusion of $\sim 2$ to $4 \%$ at $\mathrm{MeV}$ to $\mathrm{GeV}$ energies, which are lower than the $\mathrm{TeV}$ energies considered here. The relatively small ratio of perpendicular to parallel diffusion of $\sim 0.3 \%$ may be a result of lower turbulence levels in the LIC compared to other portions of the galaxy.

In our second scenario for the cosmic-ray anisotropy, we consider the contributions of supernova remnants (SNRs) to the local cosmic-ray gradient (43-48). A statistical model of cosmic rays originating from SNR sources based on the temporal and spatial distributions of supernova sources (43) suggests that cosmic rays are injected locally by different sources and time dependently diffuse throughout the galaxy. In the case of the convective anisotropy (supplementary text, section 2), the cosmic-ray gradient is created by steady cosmic-ray diffusion against the interstellar flow. Therefore, the cosmic-ray gradient driven by SNRs depends on the time and spatial distribution of SNR sources. Because parallel diffusion in the LISM is far more rapid than perpendicular diffusion, cosmic rays have a strong tendency to stream along the interstellar magnetic field. Provided that the GCR density gradient along the LISM magnetic field has the same sign as that of the convective gradient and that the cosmic-ray density gradient produces a maximum anisotropy magnitude similar to that in observations, the anisotropy associated with diffusion from SNR sources has a global morphology similar to that of the convective anisotropy (supplementary text, section 5). That is, the dominance of parallel diffusion in the LISM can result in a similar global morphology for both the convective anisotropy and that driven by SNR sources.

We developed a model of the LISM magnetic field that is deflected around the heliosphere (supplementary text, section 3) and analyzed its influence on high-energy ( $\mathrm{TeV}$ ) cosmic-ray anisotropies. We constructed a sky map (Fig. 2) of cosmic-ray flux as viewed from the Sun, using Monte-Carlo calculations of $10^{4}$ individual cosmic-ray trajectories in this perturbed magnetic field structure.

General ordering about the magnetic equator deduced from the IBEX ribbon is apparent in the high-energy (TeV) cosmic-ray observations (Fig. 2, left). Some features absent in the simulation results can be attributed to the model's lack of interstellar turbulence (22) that should cause small-scale 
anisotropic structures, local GCR acceleration that may result in a more defined excess near the heliotail (21), and asymmetric influences on the heliosphere by the interstellar magnetic field (49-53). The modeled and observed anisotropies show some differences (e.g., the region from right ascension $=-30$ to $90^{\circ}$ shows weaker modeled than observed anisotropies).

That a similar ordering of the global cosmicray anisotropy maps is generated simply by taking into account IBEX observations of lowenergy interstellar neutral atoms to deduce the interstellar velocity, and the IBEX ribbon to deduce the local interstellar magnetic field direction reveals consistency with independent observations across 10 orders of magnitude in particle energy (keV energies in the IBEX ribbon compared to $10-\mathrm{TeV}$ cosmic ray energies). Thus, local interstellar conditions play a key role in ordering veryhigh-energy $(\mathrm{TeV})$ cosmic rays in the immediate interstellar environment of the Sun.

\section{References and Notes}

1. N. A. Schwadron, H. E. Spence, R. Came, Eos 92, 297 (2011).

2. D. J. McComas et al., Science 336, 1291-1293 (2012).

3. D. J. McComas et al., Science 326, 959-962 (2009).

4. N. A. Schwadron et al., Science 326, 966-968 (2009).

5. H. O. Funsten et al., Science 326, 964-966 (2009).

6. S. A. Fuselier et al., Science 326, 962-964 (2009).

7. J. Heerikhuisen et al., Astrophys. J. 708, L126-L130 (2010).

8. J. Heerikhuisen, N. V. Pogorelov, Astrophys. J. 738, 29 (2011).

9. N. A. Schwadron, D. J. McComas, Astrophys. J. 764, 92 (2013).

10. P. C. Frisch et al., Astrophys. J. 760, 106 (2012).

11. P. C. Frisch, N. A. Schwadron, http://arxiv.org/abs/ 1310.2922 (2013)
12. D. J. McComas, M. A. Dayeh, H. O. Funsten, G. Livadiotis, N. A. Schwadron, Astrophys. J. 771, 77 (2013).

13. K. Nagashima, K. Fujimoto, R. M. Jacklyn, J. Geophys. Res. 103, 17429 (1998).

14. D. L. Hall et al., J. Geophys. Res. 104, 6737 (1999).

15. G. Guillian et al., Phys. Rev. D Part. Fields Gravit. Cosmol. 75, 062003 (2007).

16. M. Amenomori et al., in Proceedings of the 9th Annual International Astrophysics Conference, AIP Conference Proceedings, ]. Le Roux, G. P. Zank, A. J. Coates, V. Florinski, Eds. (American Institute of Physics, College Park, MD, 2010), vol. 1302, p. 285.

17. M. Amenomori; The Tibet AS $\gamma$ Collaboration, Astrophys. Space Sci. Trans. 6, 49-52 (2010).

18. A. A. Abdo et al., Phys. Rev. Lett. 101, 221101 (2008).

19. A. A. Abdo et al., Astrophys. J. 698, 2121-2130 (2009).

20. R. Abbasi et al., Astrophys. J. 740, 16 (2011).

21. P. Desiati, A. Lazarian, Astrophys. J. 762, 44 (2013).

22. G. Giacinti, G. Sigl, Phys. Rev. Lett. 109, 071101 (2012).

23. R. Lallement et al., Science 307, 1447-1449 (2005).

24. M. Opher, E. C. Stone, T. I. Gombosi, Science 316, 875-878 (2007).

25. M. Witte, Astron. Astrophys. 426, 835-844 (2004).

26. E. Möbius et al., Science 326, 969-971 (2009).

27. E. Möbius et al., Astrophys. J. Suppl. Ser. 198, 11 (2012).

28. M. Bzowski et al., Astrophys. J. Suppl. Ser. 198, 12 (2012).

29. L. J. Gleeson, W. I. Axford, Astrophys. J. 154, 1011 (1968).

30. M. A. Forman, L. ]. Gleeson, Astrophys. Space Sci. 32 77-94 (1975)

31. A. Shalchi, I. Büsching, A. Lazarian, R. Schlickeiser, Astrophys. J. 725, 2117-2127 (2010).

32. I. Büsching, M. S. Potgieter, Adv. Space Res. 42 504-509 (2008)

33. V. S. Ptuskin, I. V. Moskalenko, F. C. Jones, A. W. Strong, V. N. Zirakashvili, Astrophys. J. 642, 902-916 (2006).

34. J. R. Jokipii, Science 307, 1424-1425 (2005).

35. J. R. Jokipii, Science 316, 839-840 (2007).

36. L. C. Lee, J. R. Jokipii, Astrophys. J. 206, 735 (1976).

37. A. H. Minter, S. R. Spangler, Astrophys. J. 458, 194 (1996).

38. S. Chandrasekhar, G. Munch, Astrophys. J. 115, 103 (1952).
39. J. R. Jokipii, E. N. Parker, Astrophys. J. 155, 799 (1969).

40. ]. W. Armstrong, B. J. Rickett, S. R. Spangler, Astrophys. J. 443, 209 (1995).

41. J. R. Jokipii, in Proceedings of the 2nd Guillermo Haro Conference, ]. Franco, A. Carraminana, Eds. (Cambridge Univ. Press, Cambridge, 1999), p. 70.

42. ]. Giacalone, ]. R. Jokipii, Astrophys. ]. 520, 204-214 (1999).

43. P. Blasi, E. Amato, J. Cosmol. Astropart. Phys. 2012, 011 (2012)

44. A. D. Erlykin, A. W. Wolfendale, Astropart. Phys. 25 183-194 (2006).

45. I. Büsching, M. Pohl, R. Schlickeiser, Astron. Astrophys. 377, 1056 (2001).

46. M. Pohl, D. Eichler, Astrophys. J. 766, 4 (2013).

47. I. Busching et al., Astrophys. J. 619, 314-326 (2005).

48. C. Evoli, D. Gaggero, D. Grasso, L. Maccione, Phys. Rev. Lett. 108, 211102 (2012).

49. G. P. Zank, Space Sci. Rev. 89, 413-688 (1999).

50. R. Ratkiewicz et al., Astron. Astrophys. 335, 363 (1998).

51. N. V. Pogorelov, G. P. Zank, Astrophys. J. 636, L161-L164 (2006).

52. M. Opher, E. C. Stone, P. C. Liewer, Astrophys. J. 640, L71-L74 (2006).

53. N. V. Pogorelov, ]. Heerikhuisen, G. P. Zank, S. N. Borovikov, Space Sci. Rev. 143, 31-42 (2009).

54. R. Schönrich, ]. Binney, W. Dehnen, Mon. Not. R. Astron. Soc. 403, 1829-1833 (2010).

55. H. O. Funsten et al., Astrophys. J. 776, 30 (2013).

Acknowledgments: We thank all those who made IBEX possible. IBEX is primarily funded by NASA's Explorers Program (Contract no. NNG05EC85C). IBEX data are available at http://ibex.swri.edu/researchers/publicdata.shtml. IceCube cosmic ray data are available from http://icecube.wisc.edu/science/data.

\section{Supplementary Materials}

www.sciencemag.org/content/343/6174/988/suppl/DC1

Supplementary Text

References (56-67)

21 August 2013; accepted 29 January 2014

Published online 13 February 2014;

10.1126/science. 1245026

\section{Nanoporous $\mathrm{BiVO}_{4}$ Photoanodes with Dual-Layer Oxygen Evolution Catalysts for Solar Water Splitting}

\section{Tae Woo Kim and Kyoung-Shin Choi*}

Bismuth vanadate $\left(\mathrm{BiVO}_{4}\right)$ has a band structure that is well-suited for potential use as a photoanode in solar water splitting, but it suffers from poor electron-hole separation. Here, we demonstrate that a nanoporous morphology (specific surface area of 31.8 square meters per gram) effectively suppresses bulk carrier recombination without additional doping, manifesting an electron-hole separation yield of 0.90 at 1.23 volts $(\mathrm{V})$ versus the reversible hydrogen electrode (RHE). We enhanced the propensity for surface-reaching holes to instigate water-splitting chemistry by serially applying two different oxygen evolution catalyst (OEC) layers, $\mathrm{FeOOH}$ and $\mathrm{NiOOH}$, which reduces interface recombination at the $\mathrm{BiVO}_{4} / \mathrm{OEC}$ junction while creating a more favorable Helmholtz layer potential drop at the OEC/electrolyte junction. The resulting $\mathrm{BiVO}_{4} / \mathrm{FeOOH} / \mathrm{NiOOH}$ photoanode achieves a photocurrent density of 2.73 milliamps per square centimenter at a potential as low as $0.6 \mathrm{~V}$ versus RHE.

$\mathrm{N}$ -type bismuth vanadate $\left(\mathrm{BiVO}_{4}\right)$ has recently emerged as a promising photoanode for use in water-splitting photoelectrochemical cells because it absorbs a substantial portion of the visible spectrum (bandgap energy, $\sim 2.4 \mathrm{eV}$ ) and has a favorable conduction band (CB) edge position very near the thermodynamic $\mathrm{H}_{2}$ evolution potential $(1,2)$. However, the solarto-hydrogen (STH) conversion efficiency achieved with $\mathrm{BiVO}_{4}$ to date has been far below what is expected because the material suffers from poor electron-hole separation yield $\left(\phi_{\text {sep }}\right)(2-6)$. Previous efforts to improve the $\phi_{\text {sep }}$ of $\mathrm{BiVO}_{4}$ mainly focused on doping studies, which were intended to improve its poor electron transport properties $(2,6-12)$

Here, we demonstrate that a high-surface-area, nanoporous $\mathrm{BiVO}_{4}$ electrode composed of particles smaller than its hole diffusion length can effectively increase $\phi_{\text {sep }}$ without additional doping. Furthermore, we investigated the effect of an oxygen evolution catalyst (OEC) layer on the interfacial recombination at the $\mathrm{BiVO}_{4} / \mathrm{OEC}$ junction, water oxidation kinetics, and the Helmholtz layer potential drop at the $\mathrm{OEC} / \mathrm{electrolyte}$ junction using two different OECs, $\mathrm{FeOOH}$ and $\mathrm{NiOOH}$. Our understanding of the $\mathrm{BiVO}_{4} / \mathrm{OEC} /$ electrolyte junction resulted in the development of a new strategy to serially apply dual layers of OECs that can optimize both the $\mathrm{BiVO}_{4} / \mathrm{OEC}$ and the $\mathrm{OEC} /$ electrolyte junctions simultaneously, enabling efficient utilization of surface-reaching holes for solar water oxidation.

Department of Chemistry, University of Wisconsin-Madison, Madison, WI 53706, USA.

*Corresponding author. E-mail: kschoi@chem.wisc.edu 
N. A. Schwadron et al.

Science 343, 988 (2014);

DOI: $10.1126 /$ science. 1245026

This copy is for your personal, non-commercial use only.

If you wish to distribute this article to others, you can order high-quality copies for your colleagues, clients, or customers by clicking here.

Permission to republish or repurpose articles or portions of articles can be obtained by following the guidelines here.

The following resources related to this article are available online at www.sciencemag.org (this information is current as of February 27, 2015 ):

Updated information and services, including high-resolution figures, can be found in the online version of this article at:

http://www.sciencemag.org/content/343/6174/988.full.html

Supporting Online Material can be found at:

http://www.sciencemag.org/content/suppl/2014/02/12/science.1245026.DC1.html

This article cites 63 articles, 11 of which can be accessed free:

http://www.sciencemag.org/content/343/6174/988.full.html\#ref-list-1

This article appears in the following subject collections:

Astronomy

http://www.sciencemag.org/cgi/collection/astronomy 